\title{
Germination, Protuberance, Biphasic: Pleomorphic Adenoma
}

\author{
Anubha Bajaj* \\ Department of Pathology, Panjab University, India \\ *Corresponding author: Anubha Bajaj, Histopathologist, Punjab University, India \\ submission: August 10, 2018; Published: August 16, 2018
}

Abbreviations: CEA: Carcino Embryonic Antigen; EMA: Epithelial Membrane Antigen; GCFDP: Gross Cystic Fluid Disease Protein; PCNA: Proliferating

Cell Nuclear Antigen; Ag NOR: Argyrophilic Nucleolar Organizer Region

\section{Preface}

Pleomorphic adenoma is a ubiquitous, benign salivary gland tumour arising in the parotid besides the submandibular, sublingual and minor salivary glands. The tumours predominantly manifest between 30 to 60years as a painless, slowly-evolving lump. Precipitous enlargement, pain, facial nerve paresis or adherence of superimposed skin implicates malignant conversion. Evolution into a carcinoma ex pleomorphic adenoma may be witnessed in 3-4\% lesions [1].

\section{Delibration}

The benign, biphasic neoplasm exists in the parotid (60-70\%), the submandibular (40-70\%) and the minor salivary glands (40$70 \%$ ). The superficial lobe is implicated in $80 \%$ of the tumours where as $20 \%$ emanate from the deep lobe of the parotid. A female predominance is noted with a female to male ratio of $1.4-1.7: 1$. The consistency of the tumour is contingent to the stromal component. It varies from soft to cystic and boggy in the mucinous tumours or hard, with a glistening cut surface in the tumours with an extensive cartilaginous or chondroid component. The predilection and the management of recurrent pleomorphic adenoma lacks standardization.

\section{Morphogenesis}

The histology reveals that a capsule is focally deficient in the pleomorphic adenoma [1]. The partial and delicate pseudo-capsule permits the pseudopodia and satellite tumours to penetrate the capsule and the tumour may herniate. The recurrence of parotid adenoma surmises that microscopic lobules or pseudopodia permeate the tumour sheath. Enucleation probably dissociates the tumour nodules which may remain in the normal salivary gland. A specific presumption is an unequivocal "tumour spill" which signifies the breach of the tumour. The tumour tissue may be implanted in the surgical wound so numerous new tumours can evolve. The surgical scar is a favoured site of tumour spill. Thus, a recurrent parotid adenoma with multitudinous buds may develop (5\%). Residual disease instead of a recurrence may propound a precise terminology as the condition seems to persist/reoccur. Reoccurrence may be expressed by attempted enucleation (Figure 1). The median age of recurrent pleomorphic adenomas ranges from 33-35 years which is a decade earlier at 45-50years in patients who lack reoccurrence. The hypo-cellular and chondromyxoid pleomorphic adenomas delineate partial encapsulation, pseudopodia, satellite masses and repetitive recurrences in contrast to the hyper-cellular subtype [2].

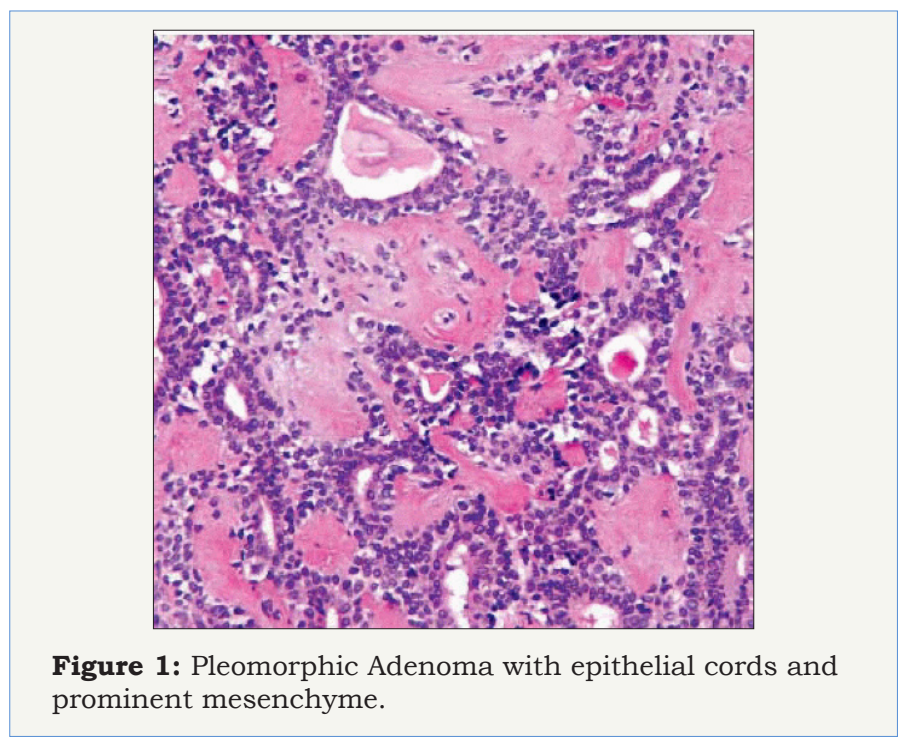

Relapses are determined by an excision biopsy for suspicious lymph nodes or cysts, a per oral access to para-pharyngeal tumours and tumour implantation by core needle biopsy or open biopsy. Multi-centricity in pleomorphic adenoma is unusual. However, recurrent parotid adenomas are multifocal. Biological and genetic factors predispose to the reoccurrences. 


\section{Pathological Representation}

Up to 100 nodules $<1 \mathrm{~mm}$ diameter may manifest on tumour relapse which act as a nidus for further recurrences. Therefore, the tumour relapses successively following surgery. The adenoma is inclined to reappear 7 to 10 years after surgical intervention. Consequently, a median follows up of 20 years is necessitated after primary intervention [3].

\section{Histopathology}

Pleomorphic adenoma is an intricate epithelial neoplasm with admixed epithelial/myoepithelial components and a mucopolysaccharide stroma (Figure 2). The neoplasm appears biphasic due to the approximation of the epithelium with the stroma. Epithelium is preponderantly glandular with foci of squamous metaplasia. The glands are lined by an epithelium with the basal myoepithelium exhibiting cuboidal, flattened, clear, spindle or hyaline appearing cells [4]. The stroma is preponderantly fibromyxoid with specifice cartilaginous differentiation. Metamorphosis between the epithelial, myopeithlial and myxochondroid cells are evidenced due to the comparable ancestry. Epithelial and the connective tissue type mucins are elaborated from the tumour epithelium. Epithelial mucin has a high content of neutral glycoprotein and mesenchymal mucin is constituted by sulphated and non-sulphated glycosaminoglycans [5].

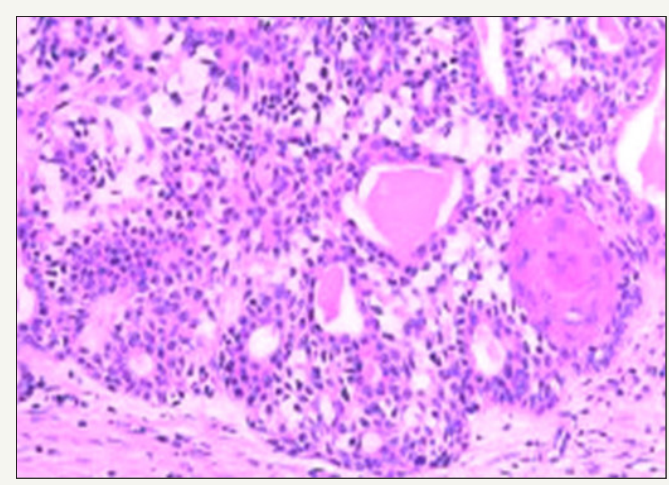

Figure 2: Cellular Myoepithelium in pleomorphic adenoma-minor salivary gland.

\section{Immunohistochemical Spectrum}

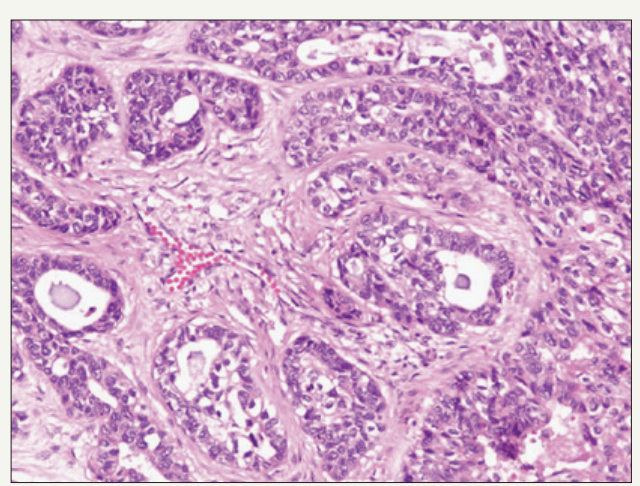

Figure 3: Malignant glandular epithelium in Pleomorphic Adenoma.
Ductal epithelium reacts to Keratin, EMA, CEA, Secretory component, lysozyme, lactoferrin, alpha-1 antitrypsin, alpha -1 antichymotrypsin, E-Cadherin, G-CDFP [6]. The keratins implicated are CK19 in the luminal cells and CK14 in the non-luminal peripheral cells. The myoepithlial component is immunoreactive for keratin, actin, myosin, smooth muscle specific antigens, p63 with fibronectin and S-100 protein expressed in the cartilaginous area [7]. Basement membrane components such as Type IV collagen and laminin are also demonstrated. The cell proliferation index is low as determined by Ki67, PCNA, AgNOR or flow cytometry (Figure 3).

Cytogenetic chromosomal translocation occurs at the $8 \mathrm{q} 12$ or 12q13-15 genetic loci. Tumour suppressor gene p53 or Tp53 is not enunciated.

\section{Malignant Metamorphosis}

An sudden enlargement of the tumour, localized pain or facial nerve paresis, evidence of previous surgical intervention or irradiation implies a malignant transformation of the tumour. The propensity is greater with the tumours located in the submandibular gland, elderly patients, enlarged tumour size, tumours exhibiting foci of hyalinization and moderate mitosis. Malignant transformation manifests dually in the epithelial and myopeithelial components. The epithelial conversion ensues as mucoepidermoid carcinoma or adenoid cystic carcinoma but can be a duct or an undifferentiated carcinoma. Immunohistochemical staining profile is identical to the benign counterpart. The monoclonal antibody B72.3 is reactive. The Tp53 tumour suppressor gene is mutated and HER 2 receptor is amplified. Genetic alterations encountered are 12q13-15 (Figure 4).

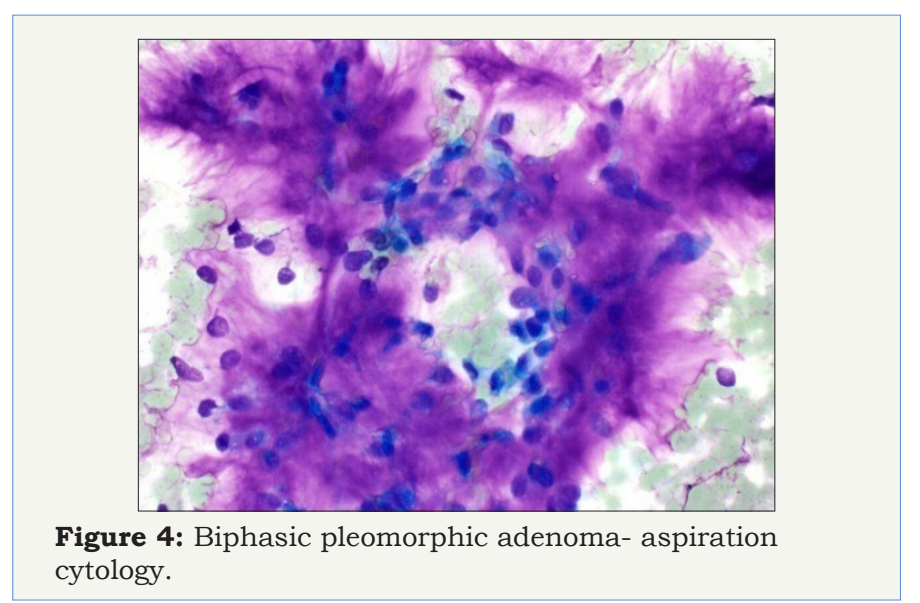

\section{Malignant Characteristics}

Are denominated as the extent of invasion beyond the capsule, minimal invasion is $<1.5 \mathrm{~cm}$ from the tumour edge, versus a wide or a frank invasion. Malignancies extending $8 \mathrm{~mm}$ or beyond the perimeter elucidate a $100 \%$ mortality [8]. A meticulous tumour sampling is a requisite. The type of the carcinoma on histology, the microscopic grade and the proliferation index are essential characteristics. Metastasis ensues in the regional lymph nodes, lungs and bones. 
A malignant variant is a biphasic composition with a duct carcinoma typifying the epithelial malignancy and the mesenchyme represented by a chondrosarcoma. A pre-existing benign tumour cannot be distinguished so it is designated as carcinoma ex pleomorphic adenoma. The de novo malignant conversion of recurrent pleomorphic adenoma varies from 0-23\%. Innumerable recurrences precede the transformation which may be accompanied by severe dysplasia (Figure 5)

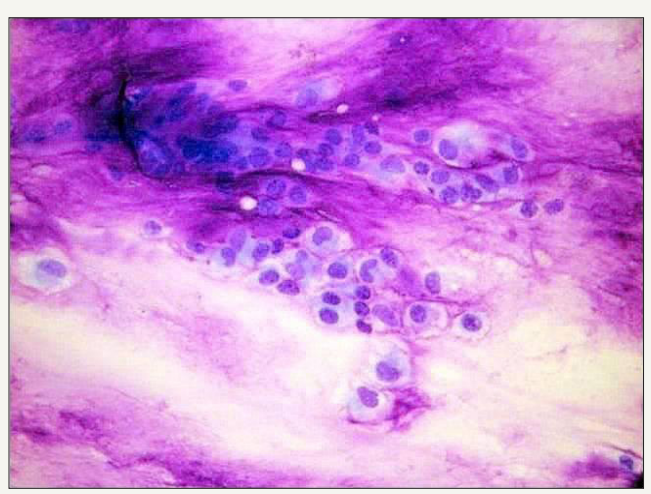

Figure 5: Myxochondroid stroma with trapped epitheliumPleomorphic adenoma cytology.

\section{Imaging of Tumour Recurrence}

The recurrent lesions are optimally evaluated by Magnetic Resonance Imaging (MRI) which demonstrates the multiple nodules, the inherent deep lobe or the para pharyngeal aspect. The magnitude of residual normal gland can also be assessed. Recurrent adenomas are delineated as round shadows of low intensity. The entire lobular architecture can be prominently delineated by the surgeon's loupe or the pathologist's microscope, in contrast to the MRI. The nodules in recurrent pleomorphic adenoma are usually in excess than are clinically apparent.

\section{Facial Nerve Outcome}

Postoperative facial nerve paresis following the primary parotid surgery develops transiently in $9 \%$ to $64 \%$ and persistently in $0-3.9 \%$ individuals. Subsequent surgeries for relapsing tumours elucidate a $90-100 \%$ transitory and an $11.3 \%-40 \%$ permanent facial paresis [9]. The facial nerve dysfunction intensifies with successive surgeries. The facial nerves sacrificed because of tumour adhesion can be restored with micro-surgical nerve grafting, usually employed in $14-30 \%$ of the tumour relapses [4]. Intra-Operative Facial Nerve Monitoring (IFNM) is utilized in parotid resections. Myography in adjunct to Intra Operative Facial Nerve Monitoring indicates that IFNM reduces the intra-operative duration, diminishes the persistent facial nerve paresis, restores the post-operative facial nerve activity.

\section{Therapeutic Options: Surgical Methodologies}

Surgical resection is the preferred treatment. The modalities of tumour resection include enucleation, partial superficial, superficial and total parotid resection, while in submandibular lesions an excision of the entire gland besides the tumour is necessitated. The pleomorphic adenomas of the minor/adjuvant salivary glands necessitate a surgical excision with a wide, uninvolved margin. The methods of parotid resection are numerous. Enucleation (which is shelling of the tumour and which disrupts the tumour capsule) along with Extra-capsular dissection (which requires scrupulous haemostasis and a limited parotid parenchyma beyond the tumour capsule) does not incise the facial nerve. Partial superficial parotid resection extirpates $2 \mathrm{~cm}$ of the parotid margin, the extent of which can increase if the facial nerve abuts the tumour. Superficial or lateral parotid resection involves the tumour eradication lateral to the facial nerve [10].

Total Parotid resection expunges the parotid tissue lateral and medial to the facial nerve. The tumour is enveloped in thin fibrous tissue with satellites and pseudopodia, thus requires an eradication in its entirety with a suitable margin of normal parenchyma to prohibit recurrences. Partial superficial parotid resection superficial, and Total Parotid resection dissect the facial nerve. Extended parotid resection dissects the facial nerve with/without the resection of the abutting structures. Minimal recurrence $(1 \%$ $4 \%$ ) ensues with a superficial parotid resection for superficial lobe lesions. Relapses with the total parotid removal manifest as $0-0.4 \%$ [2]. Pure en bloc excision is not feasible when the tumour abuts the facial nerve. However, a partial extra capsular dissection can be accomplished. A reoccurrence may ensue following a superficial parotid resection.

Nodules undergoing enucleation tend to reoccur earlier. Enucleation elucidates a $20-45 \%$ (9 times) recurrence rate in contrast to a $2-5 \%$ rate with lateral parotid resection [10]. Thus, the superficial/subtotal excision is an efficacious technique. Complete superficial parotid resection involves exposure of the main trunk and the branches of the facial nerve contiguous with the tumour. Thus, limited superficial parotid resection is considered superior for treating the primary or localized parotid neoplasm. Tumours located in the deep lobe of the parotid require a near total conservative parotid resection with probable transitory facial nerve paresis. Superficial parotid resection demonstrates minimal complications and relapses thus it is considered superior to a total parotid resection. The precise mechanism of tumour relapse is debateable [6].

Intra-operative tumour spill and inadequate initial resection are the genesis of tumour relapse. The tumour breach presents as a residual, isolated nodule or single/multiple relapses with capsular tears in $9-50 \%$ cases. Tumour reoccurrence subsequent to enucleation, extra capsular dissection or partial superficial parotid resection can be manipulated with superficial parotid or total parotid resection. The scar from the previous surgery requires elimination. Superficial parotid surgery followed by relapsing single or multiple nodules necessitate a total parotid resection. Radical and extended parotid resection is adopted for tumour penetration of the cardinal trunk or principal branches of the facial nerve. 


\section{Ramifications}

Such as Grade 2-4 mandibular marginal nerve and facial nerve paresis, skin flap necrosis and anaesthesia of ear lobules emanates from parotid resection. Marginal mandibular nerve paresis appears subsequent to submandibular gland excision. Palatal fistula arises from an excised pleomorphic adenoma of the soft palate. Precise capsular dissection is accomplished with the apposed facial nerve in order to prevent capsular rupture and neural impairment.

\section{References}

1. Zbaren P (2007) Pleomorphic adenoma of the parotid gland: Histopathological analysis of the 218 tumours. Head and Neck 29(8): 751-757.

2. Riad MA, Abdel RH, Ezzat WF, Adly A, Dessouky O, et al. (2011) Variables related to recurrence in pleomorphic adenoma: outcome of parotid surgery in 182 cases Laryngoscope 121(7): 1467-1472.

3. Patey D (1940) The treatment of mixed tumours in the parotid gland. $\mathrm{Br}$ J Surg 28(109): 29-38.
4. Redaelli ZLO, Piccioni M, Antonelli AR, Nicolai P (2008) Management and prognostic factors in recurrent parotid adenoma: personal experience and review of literature. Eur Arch Otorhinolarygol 265(4): 447-452.

5. Wallace AS, Christopher GM, Jessica MK, John WW, William MM (2013) Radiotherapy for pleomorphic adenoma. Am J Otolaryngol 34(1): 36-40.

6. Mendenhall WM, Mendenhall CM, Werning JW, Malyapa RS, Mendenhall NP (2008) Salivary gland pleomorphic adenoma. Am J Clin Oncol 31(1): 95-99.

7. Mori M, Sumitomo S, Iwai Y, Meenagham MA (1986) Immunolocalization of keratin in salivary gland pleomophic adenoma using monoclonal antibodies. Oral Surg Oral Med Oral Pathol 61(6): 611-616.

8. Rosai, Ackerman. Surgical pathology ( $11^{\text {th }}$ edn), Elseiver.

9. Robert LW, Eisele DW, Morton RP, Nicolai P, Poorten VV (2015) Etiology and management of recurrent parotid pleomorphic edenoma. Laryngoscope 125(4): 888-893.

10. Gurung U, Shrestha BL, Sinha BK, Baskota DK (2010) Pleomorphic adenoma of the salivary gland: An experience at the TUTH. Nepalese J of ENT Head and Neck Surgery 1(1): 8-11.
Creative Commons Attribution 4.0 International License

For possible submissions Click Here

\section{Submit Article}

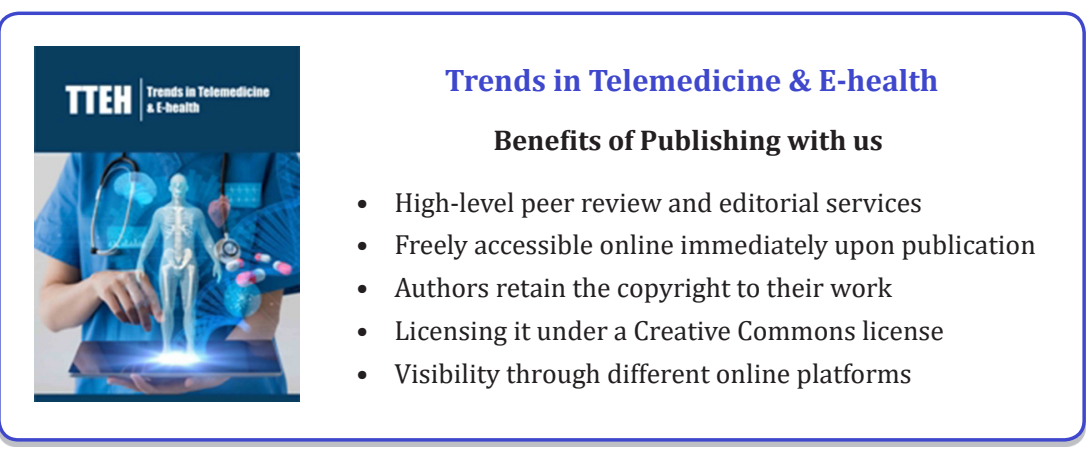

